\title{
Improvement of Power Delivery Efficiency of 11KV Power Line using Power Capacitor Placement
}

\author{
A. M. Adebimpe ${ }^{1}$, P. K. Olulope ${ }^{2}$ \\ ${ }^{1}$ Department of Mechatronics Engineering, Federal University, Oye-Ekiti (FUOYE), Oye-Ekiti, Nigeria.
${ }^{2}$ Department of Electrical and Electronic Engineering, Ekiti State University (EKSU), Ado-Ekiti, Ado-Ekiti, Nigeria
}

\begin{abstract}
Optimal capacitor placement in the distribution network is the most popular technique adopted for the control of power loss and enhancement of Volts Ampere Reactive for effective power delivery. This study, therefore, discusses improvement of distribution efficiency of $11 \mathrm{kV}$ power lines through placement of capacitor banks in the network. Power flow in the network, Ado-Ekiti $11 \mathrm{kV}$ lines, was carried out using Newton-Rapson iteration method available in MATLAB while the analysis of the network was actualized using the conventional load flow equation modeling. From the analysis of the network, voltage deviation falls within the range of -5.9 to $-180.5 \%$ which is completely outside the permissible range of $\pm 5 \%$. Voltage drop on the feeders is within the range $(1.2-1.9) \mathrm{kV}$ before and $(0.2-0.7) \mathrm{kV}$ after the reactive power of the network was compensated.
\end{abstract}

Keywords-Power Line, Distribution System, Efficiency, Reactive Power Compensation, Capacitor Banks.

\section{INTRODUCTION}

Electrical distribution networks are interconnected and meshed networks. They are arranged to be radial in operation. Network configurations may be changed by manual or automatic switching operations so that, all the loads are supplied and reduce power loss [1]. At present, with the development of power industry, power distribution network is becoming more and more complex and the power quality has also got increasing attention, which is a challenge during the operation of the distribution network. In power system, voltage and frequency are two important performance indicators, which mainly depends on the operating frequency of the system active power balance, and the reactive power balance that is mainly decided to run system voltage level [2], [3]. The control of voltage and reactive power is a major issue in power system operation [4]. Meanwhile, development of electrical power distribution system performance requires proper plans for increasing utilities efficiency. Different approaches are used to reduce losses such as optimal use of electrical equipments, optimal use of loading at the transformers, reconfiguration, and optimal capacitor placement, optimal placement of DG (Distributed Generation) and removal of harmonics. Amongst all, capacitor placement is comparatively lesser operating cost [5], [6], [7]. Reactive power is very important for the distribution network both on the economy and society .Because the equipment needs reactive power to establish field. In the process of highspeed operation of some equipment, the reactive power variation with time is quite fast. So if the reactive power consumed is not compensated in time, the security and reliability of the distribution system will be threatened which may result to voltage sag and variation that could lead to the collapse of the power system [8], [9]. Reactive power compensation is an important issue in electric power systems, involving operational, economical and quality of service aspects [10]. Placement of capacitors has been considered mainly to enhance the line voltage levels above $90 \%$ of the nominal voltage, power factor correction, and losses reduction. Power factor correction permits additional loads to be served by the existing system. That is, reactive power compensation can effectively improve power quality by reducing the line losses so as to improve the efficiency of the power network. In this way, distribution capacity of the lines is increased and the output of generator and transformer is also enhanced and the system may acquire longer life span and has greater reliability [11], [12], [13]. Capacitor placement in power network has two major concerns in it. The first one is the identification of capacitor location and the second is the amount of capacitor inclusion at the identified location. The most conventional sensitivity analysis has been followed for finding the optimal location and the conventional searching adapted in order to find the 
amount of inclusion of capacitors. For optimal placement of capacitors in distribution network, different techniques have been used by the authors in the past; the contribution made by Majid et, al [14] used Body Immune Algorithm where sensitivity analysis and ranking of the buses is carried out to know the exact position of the capacitors. Dynamic programming assuming the capacitor sizes as discrete variables adapted by Duran [15]. Artificial methods for capacitor placement include TABU search, Steel plating, Particle crow theory, Fuzzy network theory [16]. The micro genetic concepts involving enhanced genetic algorithm was proposed in [17], [18]. Optimal capacitor placement was carried out through genetic algorithm by [19]. The number of locations was considered as the total variables for genetic algorithm. The power flow constraints were handled through fuzzy logic concepts. Voltage control in an electrical power system is important for proper operation of electrical power equipment to prevent damage such as overheating of generators and motors, to reduce distribution line losses and to maintain the ability of the system to withstand and prevent voltage collapse [20], [21]. In general terms, reactive power compensation is essential for safe and economical operation of distribution network, related to whether the user can get the safety and quality of electric energy. The distribution network is directly connected with the load, the reactive power consumed by the line and the load must be balanced, otherwise it will affect the operation level of the voltage. So, research on reactive compensation technology for power distribution systems plays a significant role in safe operation of the distribution network and the improvement of the economic benefit of the power grid. The studied power network is fed from 1x15MVA \& $1 \times 7.5 \mathrm{MVA} 33 / 11 \mathrm{kV}$ distribution station through four $11 \mathrm{kV}$ feeders. The feeders are: Okesa, Basiri, Ajilosun and Adebayo. At the load points, 17 (50kVA), 43 (100kVA), 28 (200kVA), 32 (300kVA), 6 (315kVA), 57 (500kVA) and 1 (750kVA) distribution transformers further reduce the voltage from $11 \mathrm{kV}$ to $415 \mathrm{~V}$ for customers' consumption.

\section{IMPEDANCE MODELING OF THE FEEDERS}

\section{Okesa Feeder}

The ratings of Distribution Transformers on Okesa Feeder are: 50kVA, 100kVA, 200kVA, 300kVA, 315kVA and $500 \mathrm{kVA}$. The current ratings are as follows:

$$
\mathrm{P}=\sqrt{3} \mathrm{VI}(\mathrm{kVA})
$$

where $\mathrm{V}$ is Voltage and $\mathrm{I}$ is Current.

For 50kVA transformer;

$$
\begin{aligned}
& 50=\sqrt{3} \times 11 \times I \\
& I=\frac{50}{\sqrt{3} \times 11}=2.62 A
\end{aligned}
$$

For 100kVA transformer;

$100=\sqrt{3} \times 11 \times \mathrm{I}$

$\mathrm{I}=\frac{100}{\sqrt{3} \times 11}=5.25 \mathrm{~A}$

200kVA transformer;

$200=\sqrt{3} \times 11 \times \mathrm{I}$

$\mathrm{I}=\frac{200}{\sqrt{3} \times 11}=10.50 \mathrm{~A}$

300kVA transformer;

$300=\sqrt{3} \times 11 \times \mathrm{I}$

$I=\frac{300}{\sqrt{3} \times 11}=15.75$

$315 \mathrm{kVA}$ transformer;

$315=\sqrt{3} \times 11 \times \mathrm{I}$

$\mathrm{I}=\frac{315}{\sqrt{3} \times 11}=16.54 \mathrm{~A}$

For 500kVA transformer;

$500=\sqrt{3} \times 11 \times \mathrm{I}$

$\mathrm{I}=\frac{500}{\sqrt{3} \times 11}=26.25 \mathrm{~A}$.

(b) Tee offs Load Values

The Load values of Tee offs on Okesa Feeder, using Distribution Transformer Current Ratings are:

$\mathrm{T}_{1}=15.75+26.25=42 \mathrm{~A}$

$\mathrm{T}_{2}=26.25 \mathrm{~A}$

$\mathrm{T}_{3}=16.54+26.25+5.25+5.25=53.29 \mathrm{~A}$

$\mathrm{T}_{4}=26.25+26.25+2.62+2.62=57.74 \mathrm{~A}$

$\mathrm{T}_{5}=10.50+10.50=21.0 \mathrm{~A}$

$\mathrm{T}_{6}=15.75+10.50=26.26 \mathrm{~A}$

Total current (I) $=226.53 \mathrm{~A}$

(c) Conductor Parameters

The conductor size of Okesa, Basiri and Ajilosun Feeders is $35 \mathrm{~mm}^{2}$ while that of Adebayo Feeder is $100 \mathrm{~mm}^{2}$.

or Aluminum conductor of $35 \mathrm{~mm}^{2}$,

$\mathrm{r}_{0}=\frac{\rho}{\mathrm{q}}$

Where $r_{0}$ is resistance per kilometer, $\rho$ is Resistivity and $q$ is Conductor diameter. Therefore,

$\mathrm{r}_{0}=\frac{28}{33}=0.85 \Omega / \mathrm{km}$ while $\mathrm{x}=0.34 \Omega / \mathrm{km}$ (from Electrical Cable Catalogue).

$\mathrm{R}(17.7 \mathrm{~km})=17.7 \times 0.85=15.05 \Omega$

$\mathrm{X}(17.7 \mathrm{~km})=17.7 \times 0.34=6.02 \Omega$

(d) Voltage Drop Calculation

Voltage Drop calculation was done using the following load conditions:

(i) Total load lumped at the end of the line 
(ii) Half feeder load at the middle and half at the end of the line

(iii) $70 \%$ of the total load at the end of the line

(iv) $90 \%$ of the total load at the end of the line

(v) $140 \%$ of the total load at the end of the line.

(vi) Equal distribution of Feeder load.

Total Load Lumped at the end of the Line

Voltage drop calculation of Okesa feeder was done with the total feeder load lumped at the end of the line.

$$
\begin{aligned}
& =3409.28+\mathrm{j} 1363.71 \\
& =3671.91 \angle 21.8=3.67 \mathrm{kV} \\
& \qquad \mathrm{V}_{1}=\mathrm{V}_{0}-\Delta \mathrm{V}_{01} \\
& =11.55-3.67=7.88 \mathrm{kV}
\end{aligned}
$$

(ii) Half Feeder Load at the middle and half at the end of the Line

The total feeder load was divided to two equal parts and one half placed at the middle while the other half was placed at the end of the line. When the loads were in these positions, the voltage drop was calculated. The impedance diagram is as in Figure 2.1.

$$
\begin{aligned}
& \mathrm{R}_{01}=17.7 \times 0.85=15.05 \Omega \\
& \mathrm{X}_{01}=17.7 \times 0.34=6.02 \Omega \\
& \mathrm{V}_{0}=11 \times 1.05=11.55 \mathrm{kV} \quad \Delta \mathrm{V}_{01}=\mathrm{I}_{01}\left(\mathrm{R}_{01}+\right. \\
& \left.\mathrm{jX}_{01}\right) \\
& \quad=226.53(15.05+\mathrm{j} 6.02)
\end{aligned}
$$

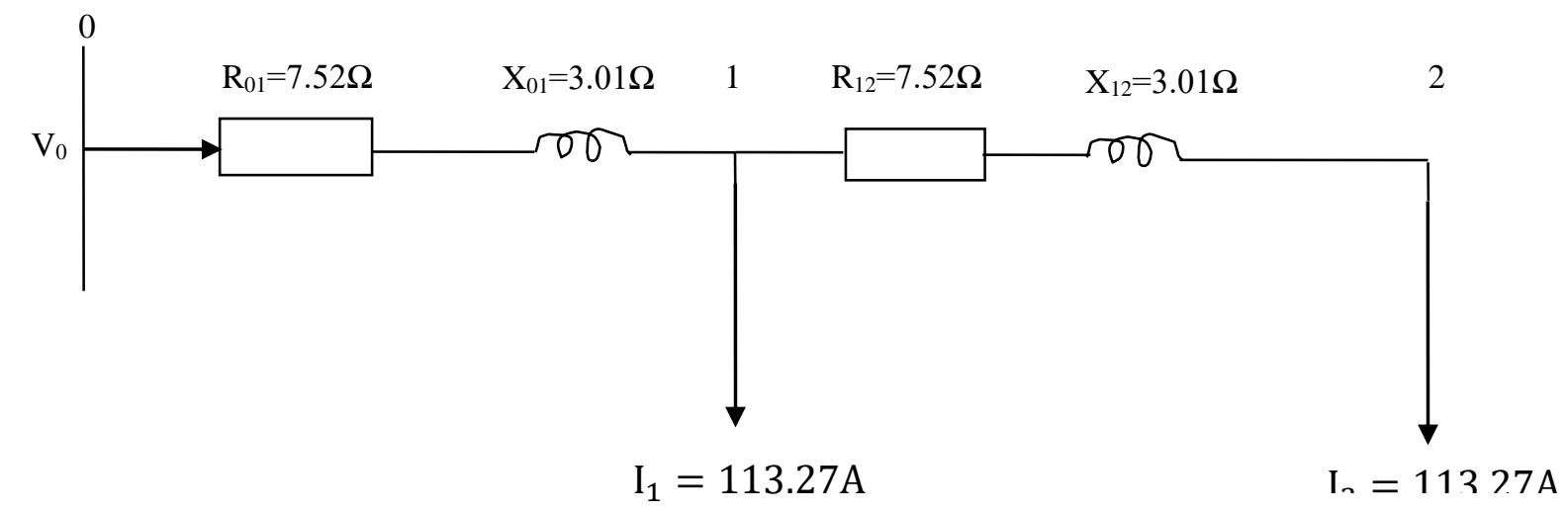

Fig.2.1: Impedance diagram of Okesa Feeder when halffeeder load is at the middle and the other half is at the end of the line.

$$
\begin{aligned}
& \mathrm{R}_{01}= \mathrm{R}_{12}=8.85 \times 0.85=7.52 \Omega \\
& \mathrm{X}_{01}=\mathrm{X}_{12}=8.85 \times 0.34=3.01 \Omega \\
& \Delta \mathrm{V}_{01}= \mathrm{I}_{01}\left[\mathrm{R}_{01}+\mathrm{j} \mathrm{X}_{01}\right] \\
&= 113.27[7.52+\mathrm{j} 3.01] \\
&= 851.79+\mathrm{j} 340.94 \\
&= 917.49 \angle 21.8 \\
&= 0.917 \mathrm{kV} \quad \\
& \mathrm{V}_{1}=\mathrm{V}_{0}-\Delta \mathrm{V}_{01} \\
&=11.55-0.917=10.63 \mathrm{kV} \\
& \begin{array}{r}
\quad \mathrm{V}_{12}=\mathrm{I}_{12}\left[\mathrm{R}_{12}+\mathrm{jX} \mathrm{X}_{12}\right] \\
=113.27[7.5 \\
=0.917 \mathrm{kV}
\end{array}
\end{aligned}
$$$$
+\mathrm{j} 3.01]
$$

$$
\begin{aligned}
& \mathrm{V}_{2}=10.63-0.917 \\
& \mathrm{~V}_{2}=9.71 \mathrm{kV} \\
& \delta \mathrm{V}=\frac{9.71-11.55}{11.55}=\frac{-1.84}{11.55} \\
& =-0.1593=-16 \% .
\end{aligned}
$$

(iii) $\mathbf{7 0 \%}$ of the total load at the end of the line

Voltage drop was also calculated with $70 \%$ of the total feeder load lumped at the end of

the line. The impedance diagram is shown in Figure 2.2. $70 \%$ of $226.53=158.57$. 


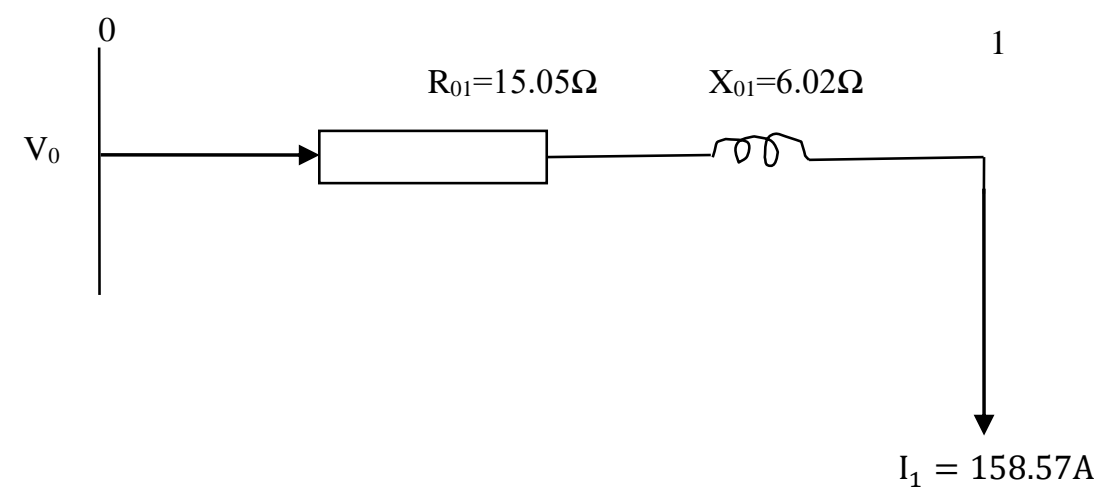

Fig.2.2: Impedance diagram of Okesa Feeder when $70 \%$ of the total load is lumped at the end of the line.

$$
\begin{gathered}
\mathrm{R}_{01}=17.7 \times 0.85=15.05 \Omega \\
\mathrm{X}_{01}=17.7 \times 0.34=6.02 \Omega \\
\mathrm{V}_{0}=11 \times 1.05=11.55 \mathrm{kV} \\
\Delta \mathrm{V}_{01}=\mathrm{I}_{01}\left[\mathrm{R}_{01}+\mathrm{j} \mathrm{X}_{01}\right] \\
=158.57(15.05+\mathrm{j} 6.02) \\
=2386.48+\mathrm{j} 954.59 \\
=2570.32 \angle 21.8=2.57 \mathrm{kV} \\
\mathrm{V}_{1}=\mathrm{V}_{0}-\Delta \mathrm{V}_{01} \\
=11.55-2.57=8.98 \mathrm{kV}
\end{gathered}
$$

$$
\begin{gathered}
\delta \mathrm{V}=\frac{\mathrm{V}-\mathrm{V}_{\mathrm{NOM}}}{\mathrm{V}_{\mathrm{NOM}}} \\
\delta V=\frac{8.98-11.55}{11.55} \\
\frac{-2.57}{11.55}=-0.2225=-22.3 \% .
\end{gathered}
$$

(iv) $90 \%$ of the total load at the end of the line Figure 2.3 shows the impedance diagram of Okesa feeder when $90 \%$ of the total load is at the end of the line.

$90 \%$ of $226.53=203.88 \mathrm{~A}$.

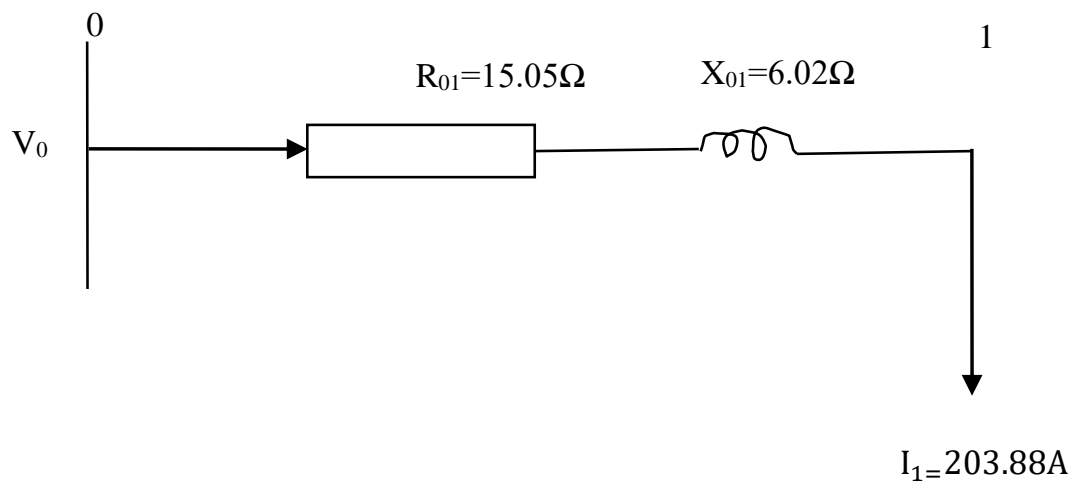

Fig.2.3: Impedance diagram of Okesa Feeder when 90\% of the total load is lumped at the end of the line.

$$
\begin{aligned}
& R_{01}=17.7 \times 0.85=15.05 \Omega \\
& X_{01}=17.7 \times 0.34=6.02 \Omega \\
& V_{0}=11 \times 1.05=11.55 \mathrm{kV} \\
& \Delta \mathrm{V}_{01}=\mathrm{I}_{01}\left(\mathrm{R}_{01}+\mathrm{j} \mathrm{X}_{01}\right) \\
& =203.88(15.05+\mathrm{j} 6.02) \\
& =3068.39+\mathrm{j} 1227.36 \\
& =3304.76 \angle 21.8=3.30 \mathrm{kV} \\
& V_{1}=V_{0}-\Delta \mathrm{V}_{01}
\end{aligned}
$$

$$
\begin{aligned}
& =11.55-3.30=8.25 \mathrm{kV} \\
\delta \mathrm{V} & =\frac{\mathrm{V}-\mathrm{V}_{\mathrm{NOM}}}{\mathrm{V}_{\mathrm{NOM}}} \\
\delta \mathrm{V} & =\frac{8.25-11.55}{11.55} \\
\frac{-3.30}{11.55} & =-0.2857=-28.6 \% .
\end{aligned}
$$

(v) $140 \%$ of the total load at the end of the line $140 \%$ of $226.53=317.14 \mathrm{~A}$ 


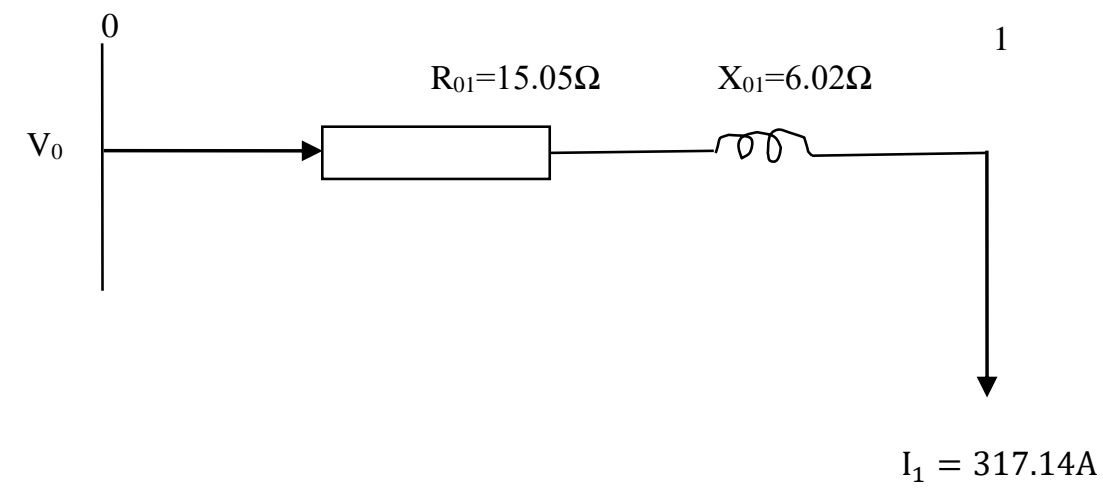

Fig.2.4: Impedance diagram of Okesa Feeder when 140\% of the total load is lumped at the end of the line.
$R_{01}=17.7 \times 0.85=15.05 \Omega$
$=11.55-5.14=6.41 \mathrm{kV}$
$X_{01}=17.7 \times 0.34=6.02 \Omega$
$V_{0}=11 \times 1.05=11.55 \mathrm{kV}$
$\Delta \mathrm{V}_{01}=\mathrm{I}_{01}\left(\mathrm{R}_{01}+\mathrm{j} \mathrm{X}_{01}\right)$
$=317.14(15.05+\mathrm{j} 6.02)$
$=4772.96+\mathrm{j} 1909.18$
$\delta V=\frac{6.41-11.55}{11.55}$
$=5140.63 \angle 21.8=5.14 \mathrm{kV}$
$\frac{-5.14}{11.55}=-0.4450=-44.5 \%$.
$V_{1}=V_{0}-\Delta \mathrm{V}_{01}$
(vi) Equal Distribution of Feeder Load
The total feeder load was distributed equally as shown in Figure 2.5 and the voltage drop calculated.

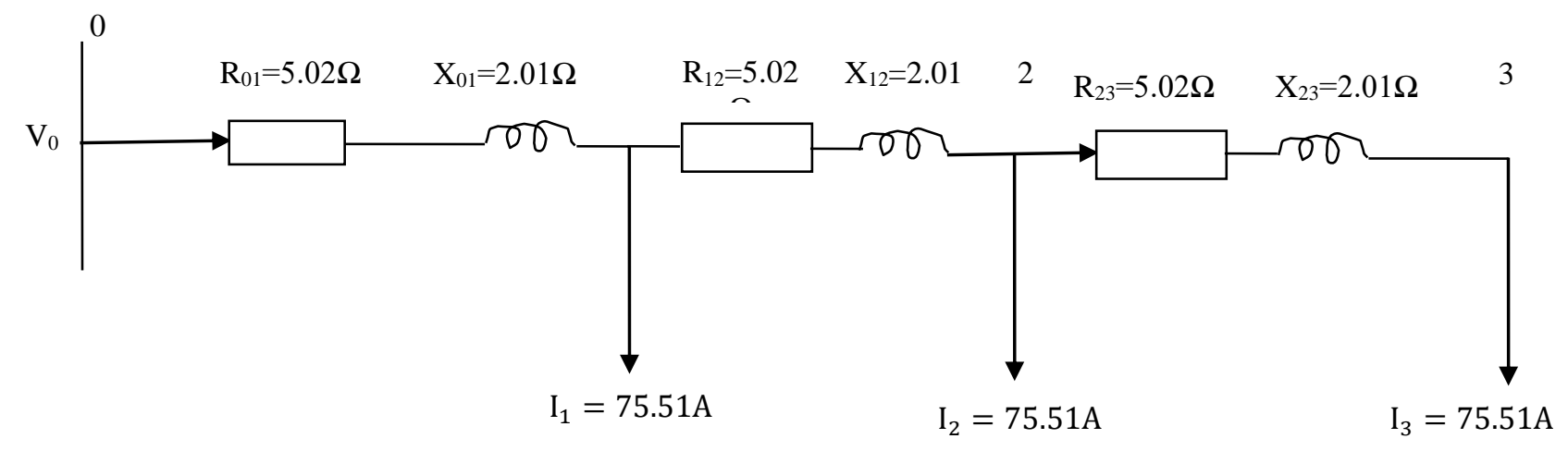

Fig.2.5: Impedance diagram of Okesa Feeder when load is distributed equally.

$$
\begin{gathered}
\mathrm{R}_{01}=\mathrm{R}_{12}=\mathrm{R}_{23}=5.9 \times 0.85 \\
=5.02 \Omega \\
\mathrm{X}_{01}=\mathrm{X}_{12}=\mathrm{X}_{23}=5.9 \times 0.34 \\
=2.01 \Omega \\
\Delta \mathrm{V}_{01}=\mathrm{I}_{01}\left(\mathrm{R}_{01}+\mathrm{j} \mathrm{X}_{01}\right) \\
=75.51(5.02+\mathrm{j} 2.01) \\
=379.06+\mathrm{j} 151.78 \\
=408.32 \angle 21.8=0.41 \mathrm{kV} \\
V_{1}=V_{0}-\Delta \mathrm{V}_{01} \\
=11.55-0.41=11.14 \mathrm{kV} \\
\Delta \mathrm{V}_{12}=\mathrm{I}_{12}\left[\mathrm{R}_{12}+\mathrm{jX}_{12}\right]=0.41 \mathrm{kV}
\end{gathered}
$$

$$
\begin{gathered}
\mathrm{V}_{2}=11.14-0.41=10.73 \mathrm{kV} \\
\text { Likewise, } \Delta \mathrm{V}_{23}=\mathrm{I}_{23}\left[\mathrm{R}_{23}+\mathrm{j} \mathrm{X}_{23}\right] \\
=0.41 \mathrm{kV} \\
\mathrm{V}_{3}=10.73-0.41=10.32 \mathrm{kV} \\
\delta V=\frac{10.32-11.55}{11.55} \\
\frac{-1.23}{11.55}=-0.1065=-10.7 \%
\end{gathered}
$$

The computation was repeated for the other feeders and the result is shown in Table 2.1. 
Table 2.1: Result of Voltage Deviation percentage computation under various possible loading conditions

\begin{tabular}{|c|c|c|c|c|}
\hline \multicolumn{2}{|c|}{ Loading Conditions } & \multicolumn{4}{|c|}{ Voltage Deviation } \\
\cline { 2 - 5 } & Okesa & Basiri & Ajilosun & Adebayo \\
\hline Total Load lumped at the end of the line & -31.8 & -37.3 & -128.9 & -17.8 \\
\hline Half Feeder Load at the middle and half at the end of the line & -16.0 & -18.7 & -64.4 & -9.0 \\
\hline $70 \%$ of the total load at the end of the line & -22.3 & -26.1 & -90.2 & -12.4 \\
\hline $90 \%$ of the total load at the end of the line & -28.6 & -33.6 & -116.0 & -16.1 \\
\hline $140 \%$ of the total load at the end of the line & -44.5 & -52.3 & -180.5 & -24.9 \\
\hline Equal Distribution of Feeder Load & -10.7 & -12.5 & -42.8 & -5.9 \\
\hline
\end{tabular}

\section{MODELING AND SIMULATION OF THE NETWORK}

Impedance modeling, simulation and load flow of the $11 \mathrm{kV}$ network was carried out using MATLAB. This was done to verify the efficacy of the reinforcement technique proposed to improve the efficiency of the network. The model consists of the source (Voltage: $11 \mathrm{kV}$, Frequency: $50 \mathrm{~Hz}$ ), a swing generator (Voltage: $11 \mathrm{kV}$, Frequency: $50 \mathrm{~Hz}$ ) connected to a 15MVA transformer that steps up the voltage from $11 \mathrm{kV}$ to $33 \mathrm{kV}$. This is connected to $1 \mathrm{x} 15 \mathrm{MVA}$ and 1 x 7.5MVA

Transformers that step down the voltage from $33 \mathrm{kV}$ to $11 \mathrm{kV}$. 4, 3-phase circuit breakers of resistances $0.001 \Omega$ are then connected. Load bus 2 and $3(11 \mathrm{kV})$ connects the injection substation with the four $11 \mathrm{kV}$ feeders. The feeders has the following distributed parameter; Okesa: number of phases: 3 , resistance per unit length: 0.085 , reactance per unit length: 0.034 and route length: $17.7 \mathrm{~km}$. Basiri: number of phases: 3 , resistance per unit length: 0.084 , reactance per unit length: 0.036 and route length: $17.2 \mathrm{~km}$. Ajilosun: number of phases: 3, resistance per unit length: 0.085, reactance per unit length: 0.036 and route length: $17.3 \mathrm{~km}$. Adebayo: number of phases: 3 , resistance per unit length: 0.028 , reactance per unit length: 0.036 and route length: $9.9 \mathrm{~km}$. Load buses 2_1, 2_2, 3_1 and 3_2 are connected for measurements. $4 \times 150 \mathrm{kVA}$ distribution transformers are connected to step down the voltage from $11 \mathrm{kV}$ to $0.415 \mathrm{kV}$ and the loads of various feeders are then lumped at the end of the line. The load flow was carried out with the existing network parameters and later performed with the incorporation of the compensating capacitors at the end of each line. The voltage and reactive power profile of the load flows were compared. The models for the load flow are shown in figures below.

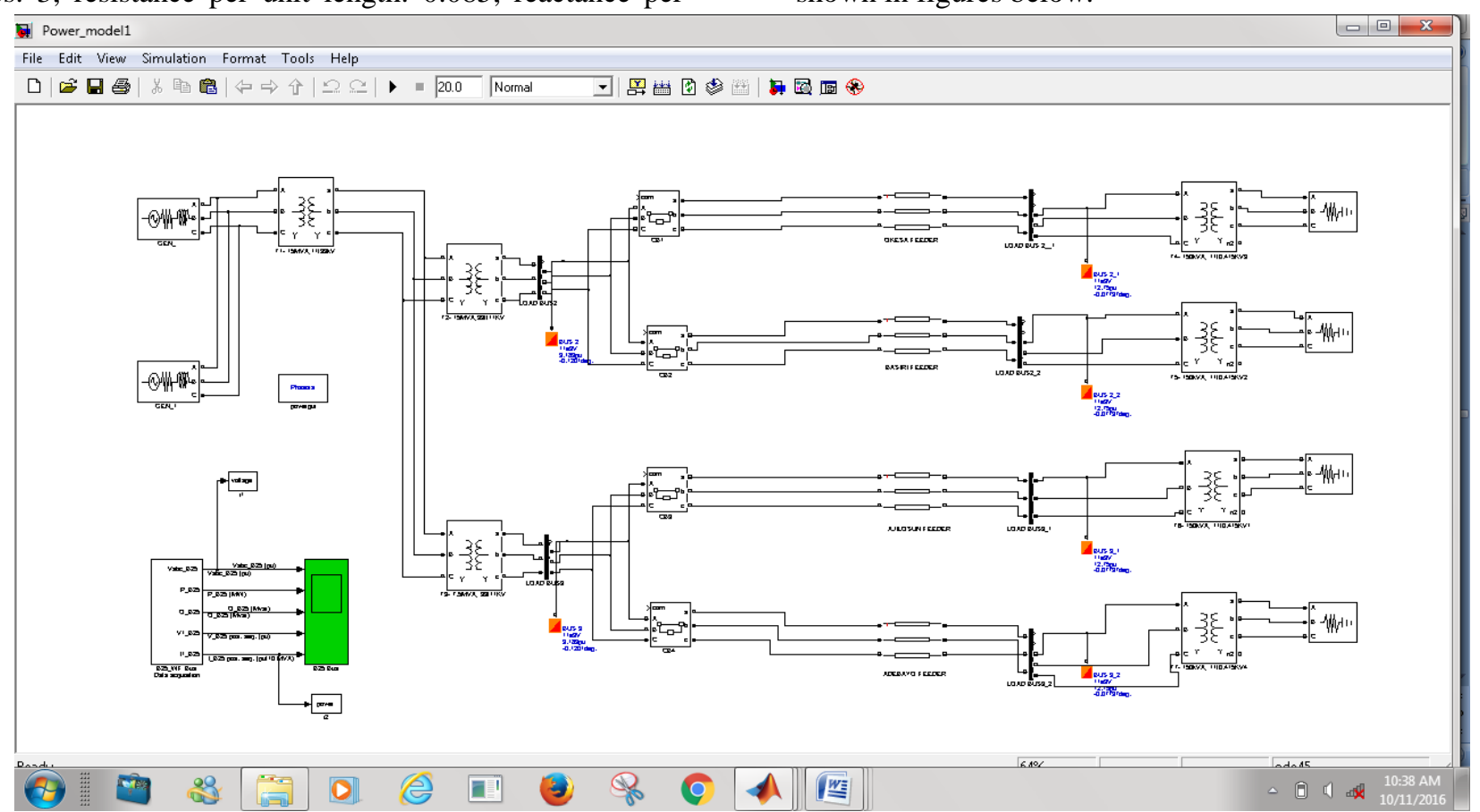

Fig.3.1: Simulation Model 1 


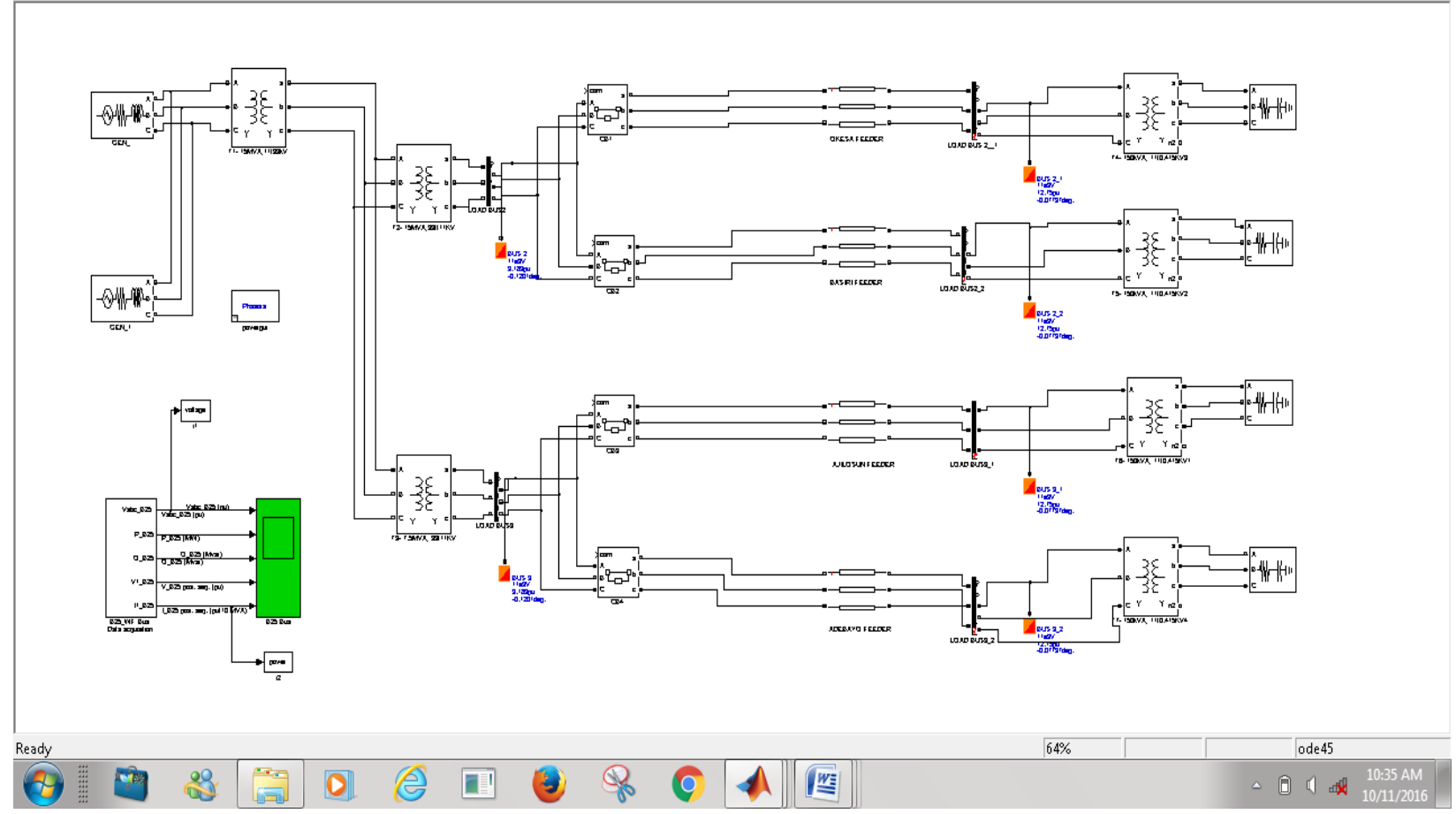

Fig.3.2: Simulation Model 2.

Result of the simulation and load flow carried out is shown below:

The Load Flow converged in 3 iterations !

SUMMARY for subnetwork No 1

Power flow result (without Improvement)

Total generation : $\mathrm{P}=1235683.75 \mathrm{~W} \mathrm{Q}=1942217.27$ var

Total PQ load : $\mathrm{P}=\quad-0.00 \mathrm{~W} \mathrm{Q}=\quad-0.00 \mathrm{var}$

Total Zshunt load : $\mathrm{P}=978499.75 \mathrm{~W} \mathrm{Q}=199492.04$ var

Total ASM load : $\mathrm{P}=0.00 \mathrm{~W} \mathrm{Q}=0.00 \mathrm{var}$

Total losses $\quad: \mathrm{P}=257184.00 \mathrm{~W} \mathrm{Q}=1742725.23$ var

$1:$ BUS $2 \mathrm{~V}=0.982 \mathrm{pu} / 11000 \mathrm{~V}-0.56 \mathrm{deg}$

Generation : $\mathrm{P}=0.00 \mathrm{~W} \mathrm{Q}=0.00$ var

PQ_load : $\mathrm{P}=0.00 \mathrm{~W} \mathrm{Q}=0.00 \mathrm{var}$

Z_shunt : $\mathrm{P}=21731.08 \mathrm{~W} \mathrm{Q}=13382.73$ var

--> *6* : $\mathrm{P}=-1014736.18 \mathrm{~W} \mathrm{Q}=-1696437.58$ var

--> BUS 2_1 : P=982724.87 W Q=1677162.80 var

--> BUS 2_2 : P=10280.24 W Q= 5892.05 var

2 : BUS 2_1 V= $0.851 \mathrm{pu} / 11000 \mathrm{~V} 3.56 \mathrm{deg}$
Generation : $\mathrm{P}=0.00 \mathrm{~W} \mathrm{Q}=0.00$ var PQ_load : $\mathrm{P}=-0.00 \mathrm{~W} \mathrm{Q}=-0.00 \mathrm{var}$ Z_shunt : $\mathrm{P}=355.40 \mathrm{~W} \mathrm{Q}=-2756.20 \mathrm{var}$ --> *3* : $\mathrm{P}=743615.22 \mathrm{~W} \mathrm{Q}=1512079.13$ var --> BUS 2 : $\mathrm{P}=-743970.62 \mathrm{~W} \mathrm{Q}=-1509322.93 \mathrm{var}$

3 : BUS 2_2 V=0.879 pu/11000V $-44.63 \mathrm{deg}$

Generation : $\mathrm{P}=0.00 \mathrm{~W} \mathrm{Q}=0.00 \mathrm{var}$

PQ_load : $P=-0.00 \mathrm{~W} Q=0.00$ var

Z_shunt : $\mathrm{P}=685.14 \mathrm{~W} \mathrm{Q}=-2681.28$ var

--> *4* $\quad: \mathrm{P}=9581.44 \mathrm{~W} \mathrm{Q}=73.97 \mathrm{var}$

--> BUS 2 : $\mathrm{P}=-10266.58 \mathrm{~W} \mathrm{Q}=2607.31 \mathrm{var}$

4 : BUS 3 V= $0.995 \mathrm{pu} / 11000 \mathrm{~V}-0.18 \mathrm{deg}$

Generation : $\mathrm{P}=0.00 \mathrm{~W} \mathrm{Q}=0.00$ var

PQ_load : $\mathrm{P}=0.00 \mathrm{~W} \mathrm{Q}=-0.00 \mathrm{var}$

Z_shunt : $\mathrm{P}=11138.54 \mathrm{~W} \mathrm{Q}=5143.55$ var

--> *6* : $\mathrm{P}=-30267.00 \mathrm{~W} \mathrm{Q}=-15080.82 \mathrm{var}$

--> BUS 3_1 : P=10501.21 W Q=6212.93 var

--> BUS 3_2 : $\mathrm{P}=8627.26 \mathrm{~W} \mathrm{Q}=3724.34 \mathrm{var}$

5 : BUS 3_1 V=0.882 pu/11000V $-44.82 \mathrm{deg}$

Generation : $\mathrm{P}=0.00 \mathrm{~W} \mathrm{Q}=0.00$ var

$\mathrm{PQ} \_$load $: \mathrm{P}=0.00 \mathrm{~W} \mathrm{Q}=0.00 \mathrm{var}$ 
$\mathrm{Z}$ shunt : $\mathrm{P}=524.96 \mathrm{~W} \mathrm{Q}=-2886.14 \mathrm{var}$ --> *2* : $\mathrm{P}=9962.07 \mathrm{~W} \mathrm{Q}=265.60 \mathrm{var}$

--> BUS $3 \quad$ : $\mathrm{P}=-10487.04 \mathrm{~W} \mathrm{Q}=2620.54$ var

6 : BUS 3_2 V=0.915 pu/11000V $-27.43 \mathrm{deg}$

Generation : $\mathrm{P}=0.00 \mathrm{~W} \mathrm{Q}=0.00$ var PQ_load : $\mathrm{P}=0.00 \mathrm{~W} \mathrm{Q}=0.00 \mathrm{var}$ $\mathrm{Z} \_$shunt $: \mathrm{P}=565.16 \mathrm{~W} \mathrm{Q}=-831.02 \mathrm{var}$ --> $* 1 * \quad: \mathrm{P}=8055.80 \mathrm{~W} \mathrm{Q}=243.44 \mathrm{var}$ --> BUS 3 : $\mathrm{P}=-8620.97 \mathrm{~W} \mathrm{Q}=587.58$ var

$7: * 1 * \mathrm{~V}=0.915 \mathrm{pu} / 415 \mathrm{~V}-27.82 \mathrm{deg}$

Generation : $\mathrm{P}=0.00 \mathrm{~W} \mathrm{Q}=0.00$ var PQ_load : $\mathrm{P}=-0.00 \mathrm{~W} \mathrm{Q}=-0.00 \mathrm{var}$ Z_shunt : $\mathrm{P}=8054.43 \mathrm{~W} \mathrm{Q}=188.27 \mathrm{var}$ --> BUS 3_2 : $\mathrm{P}=-8054.43 \mathrm{~W} \mathrm{Q}=-188.27 \mathrm{var}$

$8: * 2 * \mathrm{~V}=0.881 \mathrm{pu} / 415 \mathrm{~V}-45.35 \mathrm{deg}$

Generation : $\mathrm{P}=0.00 \mathrm{~W} \mathrm{Q}=0.00$ var PQ_load : $\mathrm{P}=-0.00 \mathrm{~W} \mathrm{Q}=0.00 \mathrm{var}$ Z_shunt $: \mathrm{P}=9959.81 \mathrm{~W} \mathrm{Q}=174.72 \mathrm{var}$ --> BUS 3_1 : P=-9959.81 W Q=-174.72 var

$9: * 3 * \mathrm{~V}=1.000 \mathrm{pu} / 11000 \mathrm{~V} 0.00 \mathrm{deg} ;$ Swing bus Generation : $\mathrm{P}=1235683.75 \mathrm{~W} \mathrm{Q}=1942217.27 \mathrm{var}$ PQ_load : $\mathrm{P}=0.00 \mathrm{~W} \mathrm{Q}=0.00 \mathrm{var}$ Z_shunt : $\mathrm{P}=22498.12 \mathrm{~W} \mathrm{Q}=22500.22 \mathrm{var}$ $->* 6 * \quad: \mathrm{P}=1213185.63 \mathrm{~W} \mathrm{Q}=1919717.05$ var

$10: * 4 * \mathrm{~V}=0.878 \mathrm{pu} / 415 \mathrm{~V}-45.02 \mathrm{deg}$ Generation : $\mathrm{P}=0.00 \mathrm{~W} \mathrm{Q}=0.00$ var PQ_load : $\mathrm{P}=0.00 \mathrm{~W} \mathrm{Q}=0.00 \mathrm{var}$ Z_shunt : $\mathrm{P}=9579.86 \mathrm{~W} \mathrm{Q}=9.63 \mathrm{var}$ --> BUS 2_2 : $\mathrm{P}=-9579.86 \mathrm{~W} \mathrm{Q}=-9.63 \mathrm{var}$

$11: * 5 * \mathrm{~V}=0.367 \mathrm{pu} / 415 \mathrm{~V}-60.24 \mathrm{deg}$

Generation : $\mathrm{P}=0.00 \mathrm{~W} \mathrm{Q}=0.00$ var PQ_load : $\mathrm{P}=0.00 \mathrm{~W} \mathrm{Q}=-0.00 \mathrm{var}$ Z_shunt : $\mathrm{P}=726246.14 \mathrm{~W} \mathrm{Q}=56.57 \mathrm{var}$ --> BUS 2_1 : $\mathrm{P}=-726246.14 \mathrm{~W} \mathrm{Q}=-56.57 \mathrm{var}$

$12: * 6 * \mathrm{~V}=0.995 \mathrm{pu} / 33000 \mathrm{~V}-0.16 \mathrm{deg}$

Generation : $\mathrm{P}=0.00 \mathrm{~W} \mathrm{Q}=0.00$ var PQ_load : $\mathrm{P}=-0.00 \mathrm{~W} \mathrm{Q}=-0.00$ var Z_shunt : $\mathrm{P}=167161.11 \mathrm{~W} \mathrm{Q}=167190.98$ var --> *5* : $\mathrm{P}=-1212880.48 \mathrm{~W} \mathrm{Q}=-1907492.43 \mathrm{var}$

--> BUS $2 \quad: \mathrm{P}=1015451.97 \mathrm{~W} \mathrm{Q}=1725204.21 \mathrm{var}$ --> BUS 3 : $\mathrm{P}=30267.41 \mathrm{~W} \mathrm{Q}=15097.24$ var
The Load Flow converged in 3 iterations!

SUMMARY for subnetwork No 2

Power flow result (with improvement)

Total generation : $\mathrm{P}=1117300.59 \mathrm{~W} \mathrm{Q}=1663373.13$ var

Total PQ load : $\mathrm{P}=\quad-0.00 \mathrm{~W} \mathrm{Q}=0.00 \mathrm{var}$

Total Zshunt load : $\mathrm{P}=1096328.46 \mathrm{~W} \mathrm{Q}=-282875.91$ var

Total ASM load : $\mathrm{P}=0.00 \mathrm{~W} \mathrm{Q}=0.00$ var

Total losses $\quad: \mathrm{P}=20972.13 \mathrm{~W} \mathrm{Q}=1946249.04 \mathrm{var}$

1 : BUS $2 \mathrm{~V}=0.986 \mathrm{pu} / 11000 \mathrm{~V}-0.50 \mathrm{deg}$ Generation : $\mathrm{P}=0.00 \mathrm{~W} \mathrm{Q}=0.00$ var PQ_load : $\mathrm{P}=-0.00 \mathrm{~W} \mathrm{Q}=-0.00 \mathrm{var}$ Z_shunt : $\mathrm{P}=21797.48 \mathrm{~W} \mathrm{Q}=-244459.54 \mathrm{var}$ --> *6* : $\mathrm{P}=-892347.89 \mathrm{~W} \mathrm{Q}=-1433651.19 \mathrm{var}$ --> BUS 2_1 : $\mathrm{P}=858013.88 \mathrm{~W} \mathrm{Q}=1674268.75 \mathrm{var}$ --> BUS 2_2 : $\mathrm{P}=12536.53 \mathrm{~W} \mathrm{Q}=3841.98 \mathrm{var}$

2 : BUS 2_1 V=0.914 pu/11000V $-2.83 \mathrm{deg}$ Generation $: \mathrm{P}=0.00 \mathrm{~W} \mathrm{Q}=0.00$ var PQ_load : $\mathrm{P}=0.00 \mathrm{~W} \mathrm{Q}=0.00 \mathrm{var}$ Z_shunt : $\mathrm{P}=453.49 \mathrm{~W} \mathrm{Q}=-224887.27 \mathrm{var}$ --> *4* : $\mathrm{P}=857372.12 \mathrm{~W} \mathrm{Q}=1743393.88$ var --> BUS $2 \quad: \mathrm{P}=-857825.61 \mathrm{~W} \mathrm{Q}=-1518506.61 \mathrm{var}$

3 : BUS 2_2 V=0.971 pu/11000V $-31.51 \mathrm{deg}$ Generation : $\mathrm{P}=0.00 \mathrm{~W} \mathrm{Q}=0.00$ var PQ_load : $\mathrm{P}=-0.00 \mathrm{~W} \mathrm{Q}=-0.00 \mathrm{var}$ Z_shunt : $\mathrm{P}=836.11 \mathrm{~W} \mathrm{Q}=-3207.51 \mathrm{var}$ $-\rightarrow * 5 * \quad: \mathrm{P}=11699.94 \mathrm{~W} \mathrm{Q}=88.53 \mathrm{var}$ --> BUS 2 : $\mathrm{P}=-12536.05 \mathrm{~W} \mathrm{Q}=3118.98$ var

4 : BUS $3 \mathrm{~V}=0.996 \mathrm{pu} / 11000 \mathrm{~V}-0.17 \mathrm{deg}$ Generation : $\mathrm{P}=0.00 \mathrm{~W} \mathrm{Q}=0.00$ var PQ_load : $\mathrm{P}=-0.00 \mathrm{~W} \mathrm{Q}=0.00 \mathrm{var}$ Z_shunt : $\mathrm{P}=11154.58 \mathrm{~W} \mathrm{Q}=4475.64 \mathrm{var}$ $->* 6 * \quad: \mathrm{P}=-34319.00 \mathrm{~W} \mathrm{Q}=-9683.45 \mathrm{var}$ --> BUS 3_1 : $\mathrm{P}=12864.15 \mathrm{~W} \mathrm{Q}=4120.27 \mathrm{var}$ --> BUS 3_2 : $\mathrm{P}=10300.27 \mathrm{~W} \mathrm{Q}=1087.54 \mathrm{var}$

5 : BUS 3_1 V=0.976 pu/11000V $-31.70 \mathrm{deg}$ Generation : $\mathrm{P}=0.00 \mathrm{~W} \mathrm{Q}=0.00$ var PQ_load : $\mathrm{P}=0.00 \mathrm{~W} \mathrm{Q}=0.00$ var Z_shunt $: \mathrm{P}=643.43 \mathrm{~W} \mathrm{Q}=-3471.71 \mathrm{var}$ $-->* 2 * \quad: \mathrm{P}=12220.22 \mathrm{~W} \mathrm{Q}=320.46 \mathrm{var}$ 


$$
\begin{aligned}
& \text {--> BUS } 3 \text { : } \mathrm{P}=-12863.65 \mathrm{~W} \mathrm{Q}=3151.25 \mathrm{var} \\
& 6 \text { : BUS 3_2 V=1.000 pu/11000V }-14.26 \mathrm{deg} \\
& \text { Generation }: \mathrm{P}=0.00 \mathrm{~W} \mathrm{Q}=0.00 \text { var } \\
& \text { PQ_load : } \mathrm{P}=-0.00 \mathrm{~W} \mathrm{Q}=0.00 \mathrm{var} \\
& \text { Z_shunt }: P=675.20 \mathrm{~W} Q=-1746.43 \text { var } \\
& \text {--> * } 1 * \quad: \mathrm{P}=9624.97 \mathrm{~W} \mathrm{Q}=287.56 \mathrm{var} \\
& \text {--> BUS } 3 \text { : } \mathrm{P}=-10300.17 \mathrm{~W} \mathrm{Q}=1458.87 \mathrm{var}
\end{aligned}
$$

$7: * 1 * \mathrm{~V}=1.000 \mathrm{pu} / 415 \mathrm{~V}-14.65 \mathrm{deg}$

Generation : $\mathrm{P}=0.00 \mathrm{~W} \mathrm{Q}=0.00$ var

PQ_load : $\mathrm{P}=0.00 \mathrm{~W} \mathrm{Q}=0.00 \mathrm{var}$

Z_shunt : $\mathrm{P}=9623.33 \mathrm{~W} \mathrm{Q}=221.65 \mathrm{var}$

--> BUS 3_2 : P=-9623.33 W Q=-221.65 var

$8: * 2 * \mathrm{~V}=0.976 \mathrm{pu} / 415 \mathrm{~V}-32.22 \mathrm{deg}$

Generation $: \mathrm{P}=0.00 \mathrm{~W} \mathrm{Q}=0.00 \mathrm{var}$

PQ_load : $\mathrm{P}=-0.00 \mathrm{~W} \mathrm{Q}=-0.00 \mathrm{var}$

Z_shunt : $\mathrm{P}=12217.45 \mathrm{~W} Q=208.99 \mathrm{var}$

--> BUS 3_1 : P=-12217.45 W Q=-208.99 var

9:*3* V=1.000 pu/11000V $0.00 \mathrm{deg}$; Swing bus

Generation : $\mathrm{P}=1117300.59 \mathrm{~W} \mathrm{Q}=1663373.13$ var

PQ_load : $\mathrm{P}=0.00 \mathrm{~W} \mathrm{Q}=0.00 \mathrm{var}$

Z_shunt : $\mathrm{P}=22498.12 \mathrm{~W} Q=22500.22$ var

--> *6* : $\mathrm{P}=1094802.47 \mathrm{~W} \mathrm{Q}=1640872.91 \mathrm{var}$

$10: * 4 * \mathrm{~V}=0.894 \mathrm{pu} / 415 \mathrm{~V}-66.64 \mathrm{deg}$

$$
\begin{aligned}
& \text { Generation : } \mathrm{P}=0.00 \mathrm{~W} \mathrm{Q}=0.00 \text { var } \\
& \text { PQ_load : } \mathrm{P}=0.00 \mathrm{~W} \mathrm{Q}=-0.00 \text { var } \\
& \text { Z_shunt : } \mathrm{P}=837345.95 \mathrm{~W} \mathrm{Q}=64.87 \text { var } \\
& \text {--> BUS 2_1 : } \mathrm{P}=-837345.95 \mathrm{~W} \mathrm{Q}=-64.87 \text { var } \\
& 11: * 5 * \mathrm{~V}=0.971 \mathrm{pu} / 415 \mathrm{~V}-31.89 \mathrm{deg} \\
& \text { Generation }: \mathrm{P}=0.00 \mathrm{~W} \mathrm{Q}=0.00 \text { var } \\
& \text { PQ_load : } \mathrm{P}=0.00 \mathrm{~W} \mathrm{Q}=0.00 \mathrm{var} \\
& \text { Z_shunt : } \mathrm{P}=11698.00 \mathrm{~W} \mathrm{Q}=9.97 \text { var } \\
& \text {--> BUS 2_2 : } \mathrm{P}=-11698.00 \mathrm{~W} \mathrm{Q}=-9.97 \mathrm{var} \\
& 12: * 6 * \mathrm{~V}=0.996 \mathrm{pu} / 33000 \mathrm{~V}-0.14 \mathrm{deg} \\
& \text { Generation }: \mathrm{P}=0.00 \mathrm{~W} \mathrm{Q}=0.00 \text { var } \\
& \text { PQ_load : } \mathrm{P}=0.00 \mathrm{~W} \mathrm{Q}=0.00 \mathrm{var} \\
& \text { Z_shunt : } \mathrm{P}=167385.30 \mathrm{~W} \mathrm{Q}=167415.21 \mathrm{var} \\
& \text {--> *3* : } \mathrm{P}=-1094572.24 \mathrm{~W} \mathrm{Q}=-1631649.43 \mathrm{var} \\
& \text {--> BUS } 2 \quad: \mathrm{P}=892867.48 \mathrm{~W} \mathrm{Q}=1454532.54 \text { var } \\
& \text {--> BUS } 3: \mathrm{P}=34319.45 \mathrm{~W} \mathrm{Q}=9701.68 \mathrm{var}
\end{aligned}
$$

When the compensating capacitors were incorporated, as shown in power flow result (summary for subnetwork No 2), Okesa receiving end voltage rose from $8.8 \mathrm{kV}$ to $10.3 \mathrm{kV}$, Basiri receiving end voltage rose from $9.6 \mathrm{kV}$ to $10.6 \mathrm{kV}$, Ajilosun's end of the line voltage rose from $9.5 \mathrm{kV}$ to $10.6 \mathrm{kV}$ while Adebayo receiving end voltage rose from $9.8 \mathrm{kV}$ to $10.8 \mathrm{kV}$.

The improvement in the voltage profile of the network is shown in Table 3.1 .

Table.3.1: Voltage Profile after Installation of Capacitor Banks

\begin{tabular}{|c|c|c|c|}
\hline FEEDER & $\begin{array}{c}\text { NOMINAL } \\
\text { VOLTAGE }(\mathrm{kV})\end{array}$ & $\begin{array}{c}\text { VOLTAGE BEFORE } \\
\text { IMPROVEMENT }(\mathrm{kV})\end{array}$ & $\begin{array}{c}\text { VOLTAGE AFTER } \\
\text { IMPROVEMENT }(\mathrm{kV})\end{array}$ \\
\hline OKESA & 11 & 8.8 & 10.3 \\
\hline BASIRI & 11 & 9.6 & 10.6 \\
\hline AJILOSUN & 11 & 9.5 & 10.6 \\
\hline ADEBAYO & 11 & 9.8 & 10.8 \\
\hline
\end{tabular}

\section{CONCLUSION}

Improvement of distribution efficiency of $11 \mathrm{kV}$ power lines through placement of capacitor banks in the network was carried out. Power flow in the studied network was carried out using Newton-Rapson iteration method available in MATLAB while the analysis of the network was actualized using the conventional load flow equation modeling. The voltage deviation of the feeders which falls within the range of -5.9 to -180.5 under various possible loading conditions is completely outside the permissible range of $\pm 5 \%$. This is an indication that the network voltage has poor quality.
Voltage drop on the feeders which was within the range (1.2 - 1.9) $\mathrm{kV}$ before and $(0.2-0.7) \mathrm{kV}$ after the reactive power compensation of the network shows that the power quality will improve considerably. This will, in turn, improve the voltage of the entire network and also solve the problem of low voltage being experienced by the consumers.

\section{REFERENCES}

[1] S. Sarkar and S. Chakravorty, "Node Voltage Improvement by Capacitor Placement in Distribution Network : A Soft Computing Approach", International 
Journal of Engineering Science and Technology, Vol. 2(10), pp 5575-5582, 2010.

[2] Y. Ma, L. Cao, X. Zhou and Z. Gao, "Research on Reactive Power Compensation Technology in Distribution Grid", 29th Chinese Control And Decision Conference (CCDC), pp 1409-1414, 2017.

[3] S. S. (Mani) Venkata and H. Rudnik, "Distribution systems: their past present and future", IEEE Power \& Energy Magazine, 7-8: 50-67, 2007.

[4] I. O. Akwukwaegbu and G. I. Okwe, "Concepts of Reactive Power Control and Voltage Stability Methods in Power System Network", Journal of Computer Engineering (IOSR-JCE), Vol 11, Issue 2, PP 15-25, (May - June 2013).

[5] T. R. Morgan, R. H. Marshall and B. J. Brinkworth, "ARES- a simulation program for the sizing and optimisation of autonomous hybrid energy systems", Solar Energy 59, pp 205-215, 1997.

[6] N. Kumar and M. Ramraj, "Combined Reconfiguration and Capacitor Placement for Distribution System Volt/Var Control through Opposition based Differential Evolution Algorithm “, Journal for Control, Measurement, Electronics, Computing and Communications, Automatika, 56:2, pp 140-148, 2015.

[7] M. Muselli, G. Notton and A. Louche, "Design of hybrid photovoltaic power generator with optimization of energy management", Solar Energy 65, pp 143-157, 1999.

[8] B. Singh and A. Chandra, "Reactive Power Compensation and Load Balancing in Electric Power Distribution System, Journal of Electrical Power \& Energy System, 102(6), pp 375-378, 1999.

[9] L. Yang and Y. Sun, "The Study for the Combination of SVG and TSC", Second International Conference of Mechanic Automation and Control Engineering, pp 3709-3711, 15-17 July 2011.

[10]N. M. Mahdi, "Power flow analysis of Rafah governorate distribution network using ETAP software", International Journal of Physical Sciences, Vol 1(2), pp 019-026, June 2013.

[11]C. H. Antunes, C. Barrico, Á. Gomes, D. F. Pires and A. G. Martin, "An Evolutionary Algorithm for Reactive Power Compensation in Radial Distribution Networks", Escola Superior de Tecnologia de Setúbal, Instituto Politécnico de Setúbal, 2910-761 Setúbal, Portugal.

[12] R. M. U. Holm, P. V. Chopade and J. Prornod,
ISSN: 2454-1311

"Optimal Placement of Capacitor for Power Loss Reduction Using ETAP Software”, ICSES' 10 International Conference on Science, Engineering \& Spirituality, 2010.

[13] A. Hemasekhar and C. Harika, "Voltage Profile Improvement by Capacitor Placement and Control in Unbalanced Distribution Systems Using Differential Evolution Algorithm", International Journal of Advanced Research in Electrical, Electronics and Instrumentation Engineering, Vol 3, Issue 8, August 2014.

[14] M. Davoodi, M. Davoudi, I. Ganjkhany, M. Arfand and and A. Aref, "Analysis of Capacitor Placement in Power Distribution Networks Using Body Immune Algorithm", Research Journal of Applied Sciences, Engineering and Technology, 4 (17), pp 3148-3153, 2012.

[15] A. Hamidat, B. Benyoucef and M. Boukadoum, "New Approach to Determine the Performances of the Photovoltaic Pumping System", Revue des Energies Renouvelables ICRESD-07 Tlemcen, 101-107, 2007.

[16] H. N. Ng, M. M. A. Salama and A.Y. Chikhani, "Classificaton of capacitor allocation techniques", IEEE Trans. Pow. Deliv., 15(1), 2000.

[17] Q. K. Nguyen, “Alternatives to grid extension for rural electrification: decentralized renewable energy technologies in Vietnam", Energy Policy 35, 25792589, 2007.

[18] B. Baran, B. J. Vallejos, R. Ramos and U. Fernandez, "Reactive power compensation using a multi-objective evolutionary algorithm", IEEE Porto Power Tech Conference, Porto, Portugal, September 2001.

[19]R. A. Prasad and E. Natarajan, "Optimization of integrated PV/wind power generation systems with battery storage", Energy 31, 1943-1954, 2006.

[20] A. Barua, "A Simulation Model for Reactive Power Compensation", International Journal for Research in Applied Science \& Engineering Technology (IJRASET), Vol 3, Issue V, 2321-9653, May 2015.

[21] J. dixon, "Reactive Power Compensation Technologies: State-of-the-Art Review", Proceedings of the IEEE, Vol 93, No.12, December 2005. 